\title{
INTRODUZIONE
}

\section{Carlo Consani}

DoI: http://dx.doi.org/10.7359/833-2017-intr

1. - La raccolta di saggi che qui si presenta trova la sua prima motivazione nel convincimento che la variazione linguistica, a qualsiasi livello la si voglia considerare, costituisce uno degli aspetti del fenomeno linguaggio più interessanti da indagare e, al tempo stesso, una sfida per quanto riguarda tanto l'individuazione delle sue motivazioni quanto il rapporto con il mutamento diacronico. Una crescente consapevolezza di tutto questo è al centro di molta della linguistica della seconda metà del '900, a partire dalla pubblicazione di due opere che continuano a configurarsi come rivoluzionarie rispetto ai paradigmi all'epoca dominanti: mi riferisco, da una parte, a Weinreich che nel suo Languages in Contact (1953) ha sperimentato una felice conciliazione tra gli assunti dello strutturalismo e quelli della ricerca dialettologica, valorizzando appieno il ruolo e il comportamento del parlante bilingue; l'altro lavoro, che pure vede in primo piano la figura Uriel Weinreich (Weinreich - Labov - Herzog 1968), richiamando l'attenzione sulla diffusione del mutamento linguistico entro una comunità di parlanti, supera di fatto alcune delle tradizionali dicotomie strutturalistiche come quella tra sincronia e diacronia e quella tra langue e parole e inaugura così un modo di affrontare lo stesso mutamento diacronico più dinamico e più sfaccettato, collegandolo decisamente con le istanze dei parlanti e della comunità linguistica.

Il cammino percorso lungo le linee inaugurate nelle opere appena evocate, che di fatto veniva a togliere molto significato ad alcuni principi dello strutturalismo saussuriano, come quello della duplice priorità del funzionamento del sistema rispetto alle realizzazioni del discorso e della sincronia sulla diacronia, è enorme: tant'è che oggi una visione variazionistica del linguaggio appare largamente accettata nella prassi e talora anche esplicitamente teorizzata, basti pensare in tal senso alla serie dei Congressi DIA che in anni recenti (2010-2016) hanno contribuito a diffondere e a riflettere sul significato, i principi e anche i limiti della variazione linguistica. 
Le circostanze più immediate che sono alla base di questa raccolta sono costituite dalle riflessioni e dagli scambi sviluppatisi attorno all'Unità di Ricerca di Chieti-Pescara costituita nell'ambito di un progetto PRIN recentemente conclusosi e che aveva come obiettivo principale la verifica della trasferibilità di alcuni assunti della moderna linguistica e sociolinguistica a fasi linguistiche del passato attestate unicamente attraverso documentazione scritta; va da sé che in tale direzione il primo e più immediato terreno di confronto riguarda appunto la verifica dell'aspetto della variazione linguistica e della variabilità dei sistemi linguistici che, se abbastanza scontato in situazioni moderne che possono valersi degli strumenti di un'adeguata raccolta di dati condotta scientificamente sul campo, appare invece problematico ricostruire per fasi attestate solo attraverso documenti scritti.

Nella gamma dei lavori raccolti coesistono situazioni appartenenti ad un passato più o meno remoto (Consani, De Rosa), situazioni contemporanee indagate attraverso raccolte di dati sul campo (Perta, Colantonio, $\mathrm{Ru}-$ scitto, Mion), nonché lavori che toccano aspetti di interesse più latamente metodologico (Canù, Ciccolone).

Affrontare l'aspetto della variazione non solo nelle dimensioni più tradizionali del discorso e dei sistemi linguistici, ma anche in riferimento al concetto di repertorio di una comunità linguistica implica di per sé chiamare in causa la componente diacronica e, con questa, la questione del mutamento linguistico; quest'ultimo aspetto, se riferito a situazioni più o meno remote come alcune di quelle appena richiamate, interessa anche la dimensione delle differenze di mezzo, una dimensione che spesso, storicamente parlando, ha comportato una configurazione di tipo diglottico dei repertori linguistici interessati: di qui tutto l'interesse, la pregnanza e anche la complessità di aggiungere al potenziale ventaglio di analisi della variazione quella relativa ai repertori linguistici.

2. - Senza pretendere di stringere nel letto di Procuste di categorie predefinite i lavori raccolti e ben cosciente della dose si arbitrarietà che quest'operazione comporta, ritengo tuttavia utile tracciare un percorso ideale che giustifica sia l'accostamento dei diversi studi sia l'interesse scientifico complessivo del volume.

Segnalo in prima istanza le implicazioni metodologiche che sono più o meno latamente connesse con i primi due contributi del volume: quello di Simone Ciccolone e quello di Silvia Canù, accomunati dal fatto di riflettere sia pure da angolature assai diverse su fenomeni generali connessi con la variabilità e il mutamento, vale a dire la natura e la collocazione del code- 
switching rispetto agli apparati formali di funzionamento del linguaggio, nel primo caso, e i processi di grammaticalizzazione, nel secondo.

Simone Ciccolone, «Estensione dello switch nella lingua incassata. Alcune osservazioni sul Matrix Language Frame», prende in esame una delle manifestazioni più tipiche della variabilità linguistica a livello di discorso del bilingue, vale a dire i fenomeni di code-switching: scopo del lavoro è quello di verificarne, attraverso l'analisi di materiale tratto da corpora di parlato, alcune limitazioni proposte nell'ambito di modelli interpretativi del code-switching, in particolare il cosiddetto modello Matrix Language Frame, enunciato da Carol Myers-Scotton negli anni '90 del secolo scorso e successivamente modificato e perfezionato dalla stessa e da altri studiosi. Dal materiale analizzato esce confermato il fatto che l'elemento critico nella formulazione dei modelli esplicativi del code-switching. è costituito dalle dimensioni delle unità coinvolte nel fenomeno: il modello MLF regge solo nel caso che le unità commutate siano limitate ad un unico elemento lessicale, mentre le inserzioni di elementi multipli pongono notevoli problemi d'analisi. Tuttavia, anche nel caso dell'inserzione di un singolo elemento, il confine fra commutazione - quindi fenomeno di contatto legato al discorso bilingue - e prestito - in quanto fenomeno che coinvolge il livello dei sistemi in contatto - rappresenta un elemento critico da determinare: da qui l'invito dell'autore a considerare la grammatica dell'enunciazione mistilingue come l'unione di diversi schemi combinatori e non come una realtà a sé stante.

Il lavoro di Silvia Canù, «Forme grammaticalizzate nel creolo di Capo Verde. Alcuni esempi dalla morfologia nominale», analizza alcuni processi di grammaticalizzazione attestati nel creolo di Capo Verde, varietà che si presenta come il frutto del contatto fra portoghese e lingue africane preesistenti; di conseguenza, oggetto dell'analisi sono alcuni processi di grammaticalizzazione (compiuti e iniziali) riscontrabili in una situazione di contatto interlinguistico: qui la variazione è colta nell'aspetto del passaggio graduale di materiale lessicale a funzioni grammaticali volte a complessificare una struttura linguistica che si presenta con caratteri affatto particolari nel panorama dei creoli, sia in virtù della durata della sua attestazione sia perché si tratta di uno dei pochi casi in cui una lingua creola è stata recentemente assunta come lingua ufficiale o coufficiale a livello statale, con tutto ciò che una simile decisione comporta.

La rassegna di casi di grammaticalizzazione attestati in questa varietà, oltre a confermare alcune tendenze universali già note, mostra che la particolare situazione di contatto che caratterizza il creolo capoverdiano non incide in maniera qualitativamente sensibile sui tipi di processi di grammaticalizzazione considerati. 
Nel mio [Carlo Consani] lavoro, «Variazione, norma e mutamento. Aspetti teorici e applicazioni al diasistema greco antico», propongo un'applicazione dei concetti della moderna linguistica della variazione ad una corpus-Sprache come il greco antico, per quanto si abbia a che fare con un corpus certo di dimensioni e caratteristiche del tutto notevoli, a cominciare dalla sua durata e dalla differenziazione diatopica. Prima di passare all'analisi dei dati del diasistema greco antico ho cercato di mostrare come, pur nella pluralità degli atteggiamenti metodologici che caratterizzano il moderno dibattito sui modelli di funzionamento del linguaggio, per lo meno a partire dalla metà del secolo scorso si sia fatto sempre più strada, anche tra linguisti di diversa estrazione, un atteggiamento che mira a uscire in qualche maniera dalle forche caudine di un sistema linguistico astratto ed esterno ai parlanti o, in altri termini, dalla competenza del parlante ideale, per apprezzare, invece, le risorse della variazione e della variabilità sincronica anche come chiave esplicativa del mutamento linguistico. Un tale complesso di presupposti teorici rivela tutta la propria validità euristica applicato a due casi opposti sul piano documentario nel panorama del greco antico, il miceneo e l'attico: nel primo caso, pure nell'estrema rarefazione e uniformità della documentazione disponibile, è possibile intravedere una modesta variazione interna che può rappresentare i prodromi dei grandi mutamenti che colpiranno il diasistema greco antico nel periodo del cosiddetto medioevo ellenico, come nel caso dell'evoluzione delle sonanti e delle labiovelari. Ben altra profondità di analisi è possibile per l'attico del periodo classico, in ragione sia della ricchezza documentaria, sia della sua varietà sia delle riflessioni metalinguistiche a noi pervenute a proposito di una siffatta situazione, che ci permettono di distinguere abbastanza accuratamente fra concreti usi linguistici adottati, le opinioni riguardo alle varietà compresenti nel repertorio e gli atteggiamenti che, più o meno scopertamente, è possibile ricostruire in proposito: una simile distinzione si rivela particolarmente appropriata nel caso di Aristofane e in quella dell'anonimo autore della cosiddetta Athenaion Politeia, che esibiscono atteggiamenti molto diversi se non opposti e un altrettanto diverso uso delle varietà del repertorio attico del V secolo. Nella parte finale dello studio, partendo dal topos della koinè ellenistica come primo vero e proprio standard del greco antico, si riflette sulle forme in cui quello che in termini moderni si definirebbe come il concetto di standard linguistico, viene lessicalizzato, per mostrare la profonda differenza tra lo standard normativo in termini moderni e la pluralità di modelli di riferimenti che invece caratterizza il greco del periodo ellenistico.

A tutt'altro ambito e tipo di fenomeni ci conduce il lavoro di Giuliano Mion, «Un arabo cipriota romanizzato? Distacchi e identità fra variazio- 
ne scrittoria e confessione religiosa», dedicato alla comunità maronita di lingua araba insediata dal medioevo nel villaggio cipriota di Kormakiti, in una situazione caratterizzata da un repertorio linguistico profondamente diverso rispetto a quello degli altri paesi arabofoni, sia per la mancanza di una norma di riferimento alta collegata con il credo musulmano (la cosiddetta fuş̧ā o arabo classico) a causa dell'adesione alla confessione maronita, sia in una situazione di estremo attrito linguistico per la pressione dell'ambiente grecofono dell'isola, che ha ridotto la varietà araba periferica parlata dalla piccola comunità a lingua domestica priva di tradizione scritta. Il lavoro analizza i recenti tentativi di acquisition planning promossi dalla rivista $O$ typos ton Maroniton e in particolare i tentativi di dotare la lingua locale di un sistema grafico diverso da quello greco, tentativi motivati da precise istanze identitarie; il confronto fra la proposta di riforma ortografica avanzata dall'arabista maltese Alexander Borg, quella tradizionale greca e le grafie spontanee che è possibile reperire sulla rete negli ultimi anni permettono all'autore di svolgere una serie di considerazioni di grande interesse, anche in prospettiva comparativa, sui collegamenti che caratterizzano le scelte del sistema scrittorio e della relativa ortografia con le istanze religiose e identitarie delle comunità interessate.

I lavori di Marika De Rosa e di Maria Loide Ruscitto si occupano di una forma particolare di variazione dei sistemi linguistici, nella fattispecie quello dell'italiano, posto in una situazione di separatezza dalla comunità linguistica di partenza. Sono noti i fenomeni che caratterizzano le lingue «emigrate», poste al centro di due diverse tendenze, l'una caratterizzata dall'impoverimento, fino all'erosione e alla scomparsa della competenza della varietà emigrata nelle generazioni successive all'evento migratorio, l'altra volta invece al mantenimento per motivi identitari del codice interessato, fino al suo artificiale recupero che può avvenire in un momento successivo l'interruzione della trasmissione intergenerazionale.

Marika De Rosa, «Tratti linguistici nelle lettere di un emigrato molisano in Canada. Fenomeni di variazione nel repertorio italiano degli anni '50», analizza un piccolo corpus di tredici lettere inedite (quattro manoscritte, nove dattiloscritte) inviate negli anni '50 del secolo scorso da un molisano originario di Ripabottoni ed emigrato in Canada, nell'intento di ricostruire una «linguistic history from below» (Elspass 2012, 156) incentrata sull'evoluzione di una varietà sociale di italiano sviluppatasi al di fuori dei tradizionali processi di standardizzazione avvenuti nella madre patria. Il basso livello di scolarizzazione del soggetto qualifica le sue produzioni, per quanto affidate alla scrittura, come particolarmente esposte a tratti dell'oralità, un'oralità favorita anche dal genere testuale della lettera e dai 
destinatari appartenenti alla ristretta cerchia familiare. L'accurata analisi linguistica cui l'autrice sottopone i testi ai diversi livelli d'analisi permette di rilevare e di precisare tutta una serie di tratti tipici dell'italiano «popolare» e, al contempo, di verificare la quasi generalizzata assenza di fenomeni da contatto con l'ambiente anglofono nel quale l'emigrante si è trovato ad operare per motivi di lavoro.

Il capitolo di Maria Loide Ruscitto, «Tra elaborazione e cristallizzazione. Il caso della comunità abruzzese emigrata a Perth (Western Australia)», è dedicato ad un diverso contesto, quello dei migranti italiani che nel secondo dopoguerra hanno scelto l'Australia come loro meta e, sulla base di dati raccolti sul campo, analizza i fenomeni connessi con la sostituzione della varietà nativa e con le modificazioni del repertorio individuale nelle prime tre generazioni di parlanti. Dopo aver passato in rassegna i diversi modelli teorici proposti per l'erosione linguistica in riferimento a contesti migratori, in cui il processo di language shift non può non essere collegato ad una situazione caratterizzata da assenza o rarefazione di stimoli linguistici nel codice di partenza, l'autrice si sofferma a delineare una situazione di vero e proprio triculturalismo in cui coesistono la cultura di partenza italiana, quella locale del paese di provenienza (Vasto), e quella del paese ospitante, dando così luogo a un complesso gioco di affermazioni di identità e di tentativi di accomodamento linguistico. Dal materiale analizzato è possibile trarre una serie dati rilevanti non solo sulle scelte di codice, e sui fenomeni di code-switching e code mixing, ma anche sulla presenza di fenomeni che sono arrivati a interessare i codici linguistici in contatto a livello di sistema, come la presenza di prestiti e di calchi.

Carmela Perta, "Variazione tra sincronia e diacronia. Aspetti del sistema morfologico di una varietà francoprovenzale», si propone di gettare un ponte tra variazione sincronica e mutamento linguistico in diacronia studiando la questione dei pronomi soggetto della varietà francoprovenzale di Faeto, per la quale è disponibile una stratificazione di dati linguistici che risale alle inchieste svolte per l'AIS (1928-1940) e, attraverso gli anni '90 del secolo scorso, arriva fino alla situazione attuale, indagata attraverso un'inchiesta linguistica sul campo che ha coinvolto un campione di 24 parlanti stratificati per età e condizione socio-economica e analizzati in due situazioni diafasiche assai diverse: il parlato spontaneo e l'attenzione metalinguistica indotta da un'artificiale situazione di traduzione di frasi dall'italiano nel codice locale. L'analisi dei dati, condotta con metodo statistico che, utilizzando la regressione categoriale, è in grado di costruire un'equazione lineare in grado di predire i valori di una variabile dipendente (l'espressione, appunto, del soggetto) sulla base dei valori delle variabili 
indipendenti attivate sia linguistiche (tipo di frase, e persona grammaticale), sia diafasiche sia sociolinguistiche; l'analisi si distingue inoltre dai precedenti analoghi studi per aver considerato distintamente anche i diversi tipi di marcatura del soggetto (semplice, con pronome debole o forte o con doppia marcatura). I risultati si presentano tutti di notevole interesse, sia là dove confermano le attese teoriche, come l'aumento della marcatura all'aumentare dell'attenzione metalinguistica, sia dove si presentano in qualche misura inattese, come nel caso della decisa marcatura del soggetto nella fascia più giovane di parlanti, in evidente contrasto con la tendenza di lungo periodo che aveva visto una decisa diminuzione nell'espressione del soggetto tra i decenni iniziali e quelli finali del secolo scorso. Al di là della pur accurata analisi descrittiva condotta, $i$ dati messi in evidenza nel lavoro possono presentare anche la base per qualche considerazione di carattere più generale, a cominciare dall'ultimo dato descritto, cioè l'inatteso aumento della marcatura del soggetto nella generazione più giovane, in evidente contrasto con lo stato recessivo della lingua minoritaria: a questo proposito ci si può chiedere se questo non possa essere visto come il riflesso delle politiche di rivitalizzazione linguistica recentemente messe in atto sia a livello nazionale (L. 482/1999) che regionale. Anche per quanto riguarda le variabili linguistiche considerate credo si debba riflettere se la costanza con cui la terza persona sia singolare che plurale è quella maggiormente esposta alla cancellazione del pronome soggetto, soprattutto nel parlato spontaneo e, in misura minore, nella traduzione di frasi, non possa riflettere lo status particolare della terza persona come «non persona» in termini benvenistiani.

Al centro del lavoro di Claudia Colantonio, «Contatto nel discorso a Gibilterra. Il caso dei marcatori discorsivi», è il repertorio linguistico di Gibilterra, che si presenta di per sé come un luogo privilegiato della variazione linguistica in virtù della sua complessità e in quanto caratterizzato da una notevole evoluzione diacronica: già a livello di sistemi linguistici, all'inglese come lingua ufficiale e allo spagnolo, nel passato lingua prima della maggior parte della popolazione della Rocca, si affianca il cosiddetto yanito, una varietà mista con tratti dei due sistemi principali, la cui natura continua tuttavia ad essere abbastanza dibattuta riguardo alla collocazione di un tale prodotto del contatto se al livello del discorso dei parlanti bilingui o a quello di un vero e proprio sistema linguistico autonomo. Il lavoro di Colantonio va ad indagare un aspetto circoscritto, ma non per questo meno interessante, di questa complessa situazione di variazione linguistica: sulla base di un piccolo corpus di interazioni semispontanee di parlanti bilingui, registrate dalla ricercatrice, vengono analizzati i marcatori discorsivi sia inglesi che spagnoli all'interno di situazioni di enunciazioni mistilingui 
ed i fenomeni di code-switching. Dall'analisi risulta generalmente confermato come la funzione più diffusa dei marcatori in un siffatto contesto plurilingue sia quella interazionale, tanto volta ad assicurare una disposizione positiva tra i parlanti, quanto impiegata per verificare l'avvenuta comprensione da parte del ricevente.

3. - La pur sintetica descrizione dei singoli lavori del volume rende possibile e opportuna qualche considerazione complessiva e conclusiva.

L'elemento più notevole che mi pare discenda dall'insieme dei lavori raccolti è che lo studio di casi profondamenti diversi, sia dal punto di vista cronologico, sia da quello tipologico, sia per la natura dei dati linguistici disponibili e sottoposti all'analisi, mostra come la variazione e le possibilità di variazione costituiscano un aspetto imprescindibile a diversi livelli: da quello del linguaggio, al livello dei sistemi linguistici analizzati, a quello del comportamento dei parlanti, nonché dei repertori individuali e comunitari. Se questo dato può considerarsi una conferma di alcune delle tendenze della ricerca linguistica della seconda metà del ' 900 richiamate all'inizio, ritengo che aver messo alla prova questo aspetto accostando situazioni affatto diverse come quelle che sono al centro dei lavori qui raccolti possa rappresentare un'ulteriore conferma della massima latina assunta qualche anno fa a titolo emblematico dell'opera di uno dei maggiori sociolinguistici europei: Variatio delectat quosdam; forse, proprio alla luce dei dati empirici offerti da questi saggi e da altre conferme della stessa natura, si potrebbe valutare l'opportunità di correggere la massima eliminando la limitazione apposta dall'oggetto indefinito.

Pescara, estate 2017

\section{RifERIMENTI BIBLIOGRAFICI}

Congresso DIA-IV 2016

Congresso DIA-IV (Universität Zürig, 12.-14. September 2016), hrsg. von M. Glessgen - J. Kabatek E. Stark - H. Völker, con indicazioni sulle precedenti edizioni dei Congressi DIA all'indirizzo: http://www. rose.uzh.ch/fr/forschung/kongresse/dia-iv-zurich.html [22.12.2016].

Dittmar 1989

N. Dittmar, Variatio delectat quosdam. Le basi della sociolinguistica, Galatina, Congedo, 1989.

Elspass 2012

S. Elspass, "The Use of Private Letters and Diaries in Sociolinguistic Investigation», in J.M. Hernández- 
Weinreich 1953

Weinreich - Labov - Herzog 1968
Campoy - C.J. Conde-Silvestre (eds.), The Handbook of Historical Sociolinguistics, Oxford, Wiley-Blackwell, 2012, 156-169.

U. Weinreich, Languages in Contact: Findings and Problems, New York, Publications of the Linguistic Circle, 1953.

U. Weinreich - W. Labov - M.I. Herzog, «Empirical Foundations for a Theory of Language Change», in W.P. Lehmann - Y. Malkiel (eds.), Directions for Historical Linguistics: A Symposium, Austin, University of Texas Press, 1968, 95-195. 
